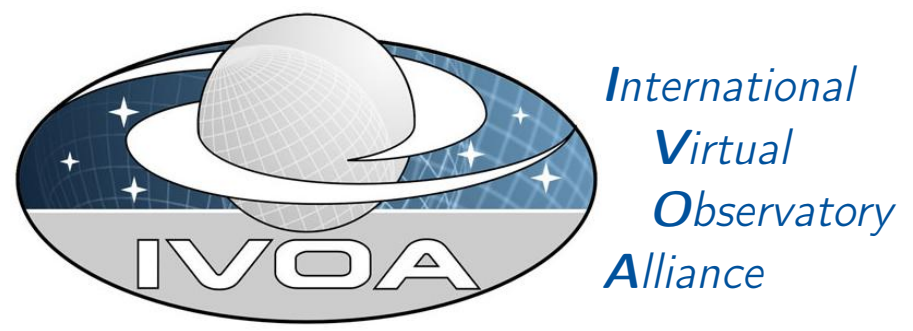

\title{
IVOA Identifiers
}

\section{Version 2.0}

\section{IVOA Recommendation 2016-05-23}

Working group

Resource Registry

This version

http://www.ivoa.net/documents/Identifiers/20160523

Latest version

http://www.ivoa.net/documents/Identifiers

Previous versions

REC-1.12

PR-20050302

PR-20040621

WD-20040209.html

WD-20031031

WD-20030930

Author(s)

PR-20030830.html

Markus Demleitner, Raymond Plante, Tony Linde, Roy Williams, Editor(s) Keith Noddle, and the IVOA Registry Working Group

Version Control

Revision 3158, 2015-11-20 09:04:09 +0100 (Fri, 20 Nov 2015)

https ://volute.g-vo.org/svn/trunk/projects/registry/Identifiers/Identifiers.tex 


\section{Abstract}

An IVOA Identifier is a globally unique name for a resource within the Virtual Observatory. This name can be used to retrieve a unique description of the resource from an IVOA-compliant registry or to identify an entity like a dataset or a protocol without dereferencing the identifier. This document describes the syntax for IVOA Identifiers as well as how they are created. The syntax has been defined to encourage global-uniqueness naturally and to maximize the freedom of resource providers to control the character content of an identifier.

\section{Status of This Document}

This document has been reviewed by IVOA Members and other interested parties, and has been endorsed by the IVOA Executive Committee as an IVOA Recommendation. It is a stable document and may be used as reference material or cited as a normative reference from another document. IVOA's role in making the Recommendation is to draw attention to the specification and to promote its widespread deployment. This enhances the functionality and interoperability inside the Astronomical Community.

A list of current IVOA Recommendations and other technical documents can be found at http://www.ivoa.net/Documents/.

\section{Contents}

1 Introduction 3

1.1 Definitions . . . . . . . . . . . . . . . . . 4

1.2 Selected Requirements . . . . . . . . . . . . . . . . 6

1.3 Rationale for Version $2 \ldots \ldots \ldots \ldots$

1.4 IVOA Identifiers within the VO Architecture $\ldots \ldots \ldots \ldots$

2 Specification 8

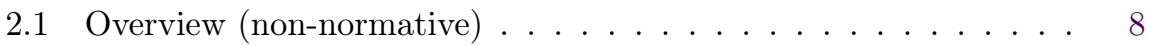

2.2 Characters . . . . . . . . . . . . . . . . . . 9

2.3 Syntax Components . . . . . . . . . . . . . . . . . . 9

2.3 .1 Scheme . . . . . . . . . . . . . . . . 9

2.3 .2 Authority . . . . . . . . . . . . . . . . 10

2.3 .3 Resource Key . . . . . . . . . . . . . . . . . . . . . 11

2.3 .4 Query . . . . . . . . . . . . . . . . . . 12

2.3 .5 Fragment . . . . . . . . . . . . . . . . 13

2.4 Usage . . . . . . . . . . . . . . . . . . . . . . . . 13

2.5 Reference Resolution . . . . . . . . . . . . . . . . . . . . . . 14

2.6 Normalization and Comparison . . . . . . . . . . . . . 14

3 Creating Identifiers 16 
4 Special Identifier Types 16

4.1 Dataset Identifiers . . . . . . . . . . . . . . . . . . . . . 16

4.2 Standard Identifiers . . . . . . . . . . . . . . . . . 17

$\begin{array}{ll}\text { A Changes from Previous Versions } & 18\end{array}$

A.1 Changes from PR-2015-07-09 . . . . . . . . . . . . . . 19

A.2 Changes from $1.12 \ldots \ldots \ldots \ldots \ldots$

A.3 Changes from v1.10 . . . . . . . . . . . . . . . . . 19

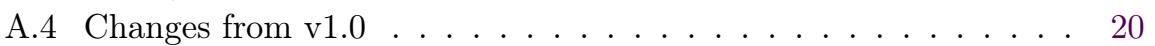

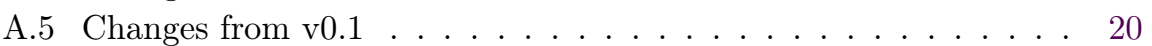

\section{Acknowledgments}

This document builds on the concept of a Uniform Resource Identifier as described in RFC 3986 (Berners-Lee et al., 2005) and its predecessors.

This document has been developed with support from the National Science Foundation's Information Technology Research Program under Cooperative Agreement AST0122449 with The Johns Hopkins University, from the UK Particle Physics and Astronomy Research Council (PPARC), from the European Commission's Sixth Framework Program via the Optical Infrared Coordination Network (OPTICON), and from the German Astrophyiscal Virtual Observatory GAVO, BMBF grant 05A14VHA.

\section{Conformance-related definitions}

The words "MUST", "SHALL", "SHOULD", "MAY", "RECOMMENDED", and "OPTIONAL" (in upper or lower case) used in this document are to be interpreted as described in RFC 2119 (Bradner, 1997).

\section{Usage of ABNF}

This specification uses ABNF (Crocker and Overell, 1997) to specify grammar rules. The rules from RFC 3986 are assumed throughout. Where both this specification and RFC 3986 define a nonterminal, the rule in this specification overrides the corresponding rule from RFC 3986.

For explicitness, we write ABNF nonterminals in angle brackets ( $\langle$ like this $\rangle$ ) throughout.

\section{Introduction}

Virtual Observatory applications frequently need to unambiguously refer to some resource or concept which is described elsewhere. It is therefore necessary 
to define global, potentially dereferenceable identifiers. In the VO, these are called IVOA identifiers (IVOIDs). An unambiguous reference within the entire Virtual Observatory requires that the identifier is globally unique. Ensuring this uniqueness inevitably requires oversight by a moderating authority; however, a flexible framework can minimize the opportunity for duplicated identifiers.

Many data providers in the $\mathrm{VO}$ were creating and using identifiers long before this specification was developed. Their choices of identifiers were made presumably to best fit the needs of the data. In order to minimize the cost of adoption of the IVOA identifier framework the design specified here maximizes the control providers have, thus allowing the reuse of the identifiers data providers already have in place as well as the creation of new idenfiers that are consistent with their overall organization.

Identifiers are crucial to the operation of registries that aid users in discovering data and services (Benson et al., 2009). In general, a registry stores descriptions of data and services in a searchable form, and it distinguishes them by the unique identifier defined here. It thus serves as a primary key for the VO Registry, and thus allows dereferencing identifiers to metadata about a resource (the resource record).

IVOA identifiers with query or fragment parts can furthermore reference essentially arbitrary entities like datasets or protocols, based on this primary mechanism of dereferencing.

We recognize that resources do not always remain in the control of a single organization forever. This necessitates a form of referencing that is locationindependent - or more precisely, organization-independent. Apart from enabling seamless transfers of data curation, an attractive use case for such identifiers is when several copies of a dataset exist at several locations around the VO and one could refer to all of them collectively, deferring the choice of a particular instance until it is actually needed. Such references thus serve as persistent pointers to data that can be flexibly resolved. This is very important to journal publishers that wish to refer to data in publications (whose useful life might be measured in decades) without worry that the references will become obsolete.

This specification, in contrast, defines organization-dependent identifiers. Persistent, organization- and location-independent identifiers are not (directly) defined here.

Referencing resources is addressed by the IETF standard for URIs, RFC 3986 (Berners-Lee et al., 2005). Thus, the framework proposed in this document builds directly on this standard. Essentially, this standard sets the parameters left open for application use by RFC 3986 .

\subsection{Definitions}

A Uniform Resource Identifier (URI) is defined by RFC 3986 as "a compact sequence of characters that identifies an abstract or physical resource" which complies with the syntax specification of that document (Berners-Lee et al., 
2005). It can point to an actual retrievable resource, but there is no requirement for it to be dereferenceable at all, let alone by a stock web browser.

An IVOA identifier, or IVOID, is a special sort of URI complying with all parts of this specification. Historically, these have also been known as IVOA Resource Names (IVORNs), in parallel to the Uniform Resource Names (URNs) that formulated extra requirements on persistency and location-independence. As plain IVOIDs do not fulfill those requirements and the term URN has been deprecated by RFC 3986, we now deprecate the term IVORN, too.

A full IVOID can thus be split into a Registry part (schema, authority, and path) and a possibly empty local part consisting of query and fragment component, again using RFC 3986 nomenclature. An IVOID with an empty local part is also known as a Registry reference.

In VO practice, the term resource is somewhat ambiguous. The IVOA Recommendation on Resource Metadata (Hanisch et al., 2007), from here on referred to as RM, defines it as "a VO element that can be described in terms of who curates or maintains it and which can be given a name and a unique identifier." It then goes on to define the relevant pieces of metadata, which later provided the foundations of the data model behind the IVOA Registry.

This might lead to the expectation that there is a 1:1 relationship between Registry records, "VO resources", and IVOA identifiers, and version 1 of this document essentially implied as much. In this version, we only require Registry references to resolve in the Registry.

IVOIDs having a nonempty local part do not dereference to Registry records. Since we want to maintain the notion that a resource is whatever a URI points to, "resource" as used here does not correspond to the usage of the term in VOResource (Plante et al., 2008). To maintain the distinction, we call resources in the sense of VOResource Registry records. These form a subset of the resources (in the URI sense) that can be referenced by IVOIDs.

We refer to organizations and providers in the sense that they are defined in $\mathrm{RM}$ :

An organization is a specific type of resource that brings people together to pursue participation in VO applications. Organizations can be hierarchical and range greatly in size and scope. At a high level, it could be a university, observatory, or government agency. At a finer level, it could be a specific scientific project, space mission, or individual researcher. A provider is an organization that makes data and/or services available to users over the network.

Definitions of other types of resources, including data collection and service, are also provided in RM, and are assumed by this document. 


\subsection{Selected Requirements}

This proposal is the result of various requirement studies for VO identifiers and registries in general (e.g. NVO ID requirements ${ }^{1}$ ). This section highlights a few of the important ones that guided the design of the ID framework.

1. A single framework should be used to identify anything a VO application can refer to, including organizations, projects (mission/telescope), data collections, and services.

2. It should be easy to compare two instances of an identifier to determine if they refer to the same object.

3. It should be possible to use an identifier to access a unique description of the resource it identifies.

4. The framework should maximize the freedom of data providers to choose identifiers for resources and collections under their control.

\subsection{Rationale for Version 2}

A need for revising the IVOA Identifiers specification was discerned ever since Gray (2012) pointed out that common practices regarding dataset identifiers were not in line with URI semantics. Also, with the publication of StandardsRegExt (Harrison et al., 2012), it became advisable to regulate the ways standards are referenced in the VO in ways compatible with the spirit of that standard.

As the Registry Working Group set about revising the Identifiers recommendation, it was decided to drop the XML representation for IVOIDs since it complicated the text but had never actually been found useful. Even in XML serializations only the URI form of IVOA identifiers had been used. Dropping the XML form nevertheless constitutes an incompatible change, which necessitates an increase in the major version number.

Despite the new major version, consensus was that current usage of IVOIDs should not be impacted and existing practices sanctioned as far as possible. Apart from deprecating the use of fragment identifiers to distinguish datasets and restricting authorities to only use $\langle$ unreserved $\rangle$ characters (which does not impact existing authority identifiers), this specification therefore refrains from modifying version 1 regulations even where they were found somewhat burdensome (e.g., as regards case-insensitiveness in resource keys).

The opportunity of a revision was also used to organize the specification content in parallel to RFC 3986; for instance, the notion of stop characters from version 1 - necessitated by the non-URI XML representation - has no counterpart in non-IVOID URIs and is now encompassed naturally by the usual rules for parsing URIs.

\footnotetext{
1 http://web.archive.org/web/20070226120639/http://nvo.ncsa.uiuc.edu/ rplante/ vo/metadata/oidreq2.txt
} 
Closely following RFC 3986 also allows rigorous definitions for the interpretation of local parts. In addition to what version 1 specified, we now allow percent-encoded characters there, and we comment on techniques to resolve IVOIDs with such local parts. This is finally used to define the standard and the dataset identifiers that started the revision process.

\subsection{IVOA Identifiers within the VO Architecture}

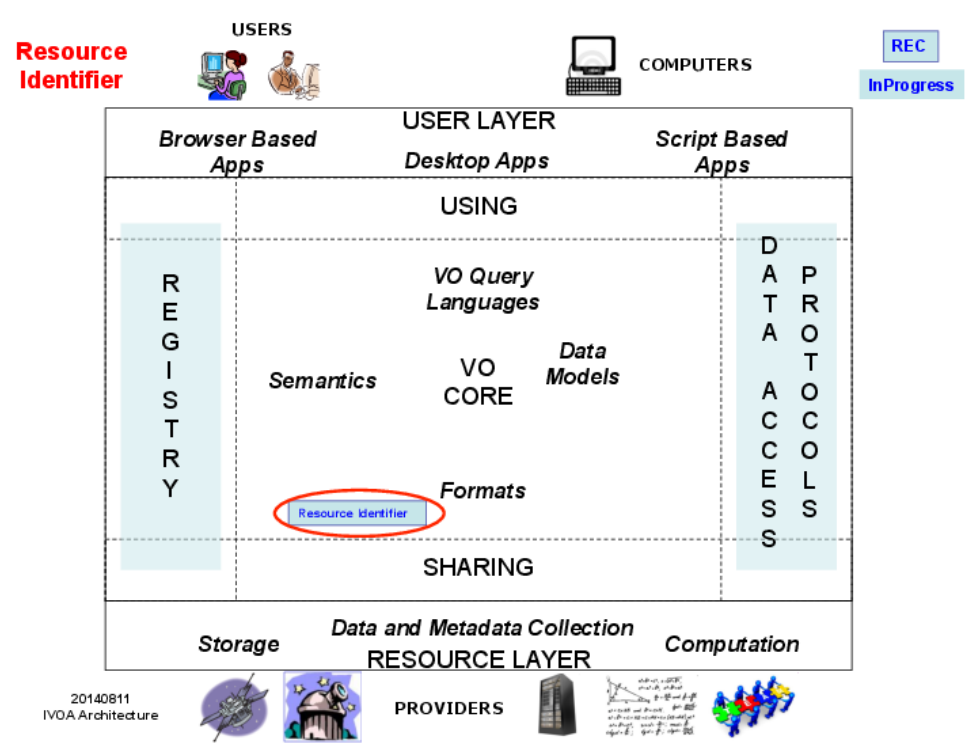

Figure 1: Architecture diagram for the IVOA Resource Identifier specification

Fig. 1 shows the role this document plays within the IVOA architecture (Arviset et al., 2010). As identifiers are the primary keys into the Registry, essentially all standards regulating the Registry depend on this specification. The data access protocols are mainly impacted through the use of dataset identifiers - e.g., in SSAP (Tody et al., 2012) and Obscore (Louys et al., 2011) -, which are also IVOID. For the same reason, VOEvent is impacted.

The core of this standard has no dependencies on other VO standards. The section on identifiers for standards depends on StandardsRegExt (Harrison et al., 2012). 


\section{Specification}

After a brief, informal specification that should be enough for non-demanding applications, this section gives, for each relevant part of RFC 3986, additional requirements for IVOA identifiers. The normative content should be read together with RFC 3986.

\subsection{Overview (non-normative)}

IVOA identifiers (IVOIDs for short) are RFC 3986-compliant URIs with a scheme of ivo. Thus, their generic form is

$$
\underbrace{\text { ivo: } / /\langle\text { authority }\rangle\langle\text { path }\rangle}_{\text {Registry part }} \underbrace{?\langle\text { query }\rangle \#\langle\text { fragment }\rangle}_{\text {local part }} \text {, }
$$

where $\langle p a t h\rangle$ is either empty or starts with a slash, and both items in the local part are optional. .

IVOIDs consisting of only scheme and authority are known as authority identifiers and play a special role in creating other IVOIDs (see sec. 3). IVOIDs without a local part must resolve to a Registry record within the IVOA Registry. Likewise, for all IVOIDs, the IVOID resulting from stripping the local part (the Registry part) must resolve within the IVOA Registry. It is called a Registry reference.

The RFC $3986\langle$ path〉 element is called resource key in IVOIDs.

Authority ids must consist of letters, numbers, dashes and dots exclusively. Resource keys must not contain URI reserved characters (essentially, only alphanumeric characters, dashes, dots, underscores, and tildes are allowed) except where an IVOA standard defines how they are to be treated.

The Registry references are, as a whole, compared case-insensitively, and must be treated case-insensitively throughout to maintain backwards compatibility with version 1 of this specification. When comparing full IVOIDs, the local part must be split off and compared preserving case, while the registry part must be compared case-insensitively.

To make IVOIDs useful where these complex rules are hard to implement (e.g., database columns), handling applications SHOULD NOT change the case of any part of IVOIDs when these might have a local part.

Examples for IVOIDs:

- ivo://ivoa.net - an IVOID without a resource key, i.e., an authority; dereferencing in the Registry must yield a vr:Authority-typed record.

- ivo://ivoa.net/std/Identifiers - an IVOID with a resource key. Dereferencing this in the Registry must yield a resource record. As long as there is no local part, an IVOID only differing in case, e.g., ivo://IVOA.NET/std/identifiers, is in every respect equivalent to it. 
- ivo://example.org/ ?path/to/\%C3\%89CLAIRE - an IVOID without guarantees as to if it resolves and what it resolves to. The Registry reference ivo://example.org/ must resolve to a valid Registry record, though.

- ivo://example.org/svc?voc.xml\#Term - an IVOID conceptually referencing some item within ivo://example.org/svc?voc.xml. If that latter IVOID can be dereferenced, there should be an entity within the resource retrieved that is itself identified by Term. The classic example would be an element with an id of Term within an XML document.

The remainder of this section contains a formalization of these points.

\subsection{Characters}

This specification poses no additional global constraints on the character content of IVOIDs over what Section 2 of RFC 3986 specifies. Special restrictions on the authority part and the resource key are given below. In particular, the $\langle$ gen-delims $\rangle$ have, where applicable, the standard URI interpretation. As IVOIDs have no use for IPv6 addresses or user components, square brackets and the commercial at sign MUST NOT occur literally in IVOIDs anywhere.

The $\langle$ sub-delims $\rangle$ MUST NOT be part of the resource key unless another IVOA specification defines their use. Their use in local parts is not restricted by this specification, nor is any semantics defined for them. Other IVOA specifications may furnish them with semantics.

In IVOIDs, characters from $\langle$ unreserved $\rangle$ MUST NOT be percent-encoded.

Percent-encoded characters are allowed in local parts (but neither in authority nor the resource key). When specifications or applications require text to be percent-encoded within an IVOID, the text MUST be encoded in UTF-8.

\subsection{Syntax Components}

\subsubsection{Scheme}

The $\langle$ scheme $\rangle$ part of IVOIDs is ivo. Note that, by RFC 3986, scheme identifiers are case-insensitive.

A URI that uses this scheme (an IVOID) signals that:

- the registry part of the IVOID and the resource it refers to have been registered in the VO Registry

- the URI complies with the additional restrictions laid down in this document

The ivo scheme does not imply a transport protocol by which the resource may be accessed. Agents, in general, should not depend on implicit mappings between IVOIDs and URIs in other schemes like http when dereferencing them. The only defined way to dereference IVOIDs is described in sect. 2.5. Resource 


\section{Note}

While the syntax for the authority identifiers allows it to look just like a DNS hostname, current convention discourages this practice to avoid the suggestion that an IVOA Identifier can be resolved like a common http URL. As of this writing, the convention of the US Virtual Astronomical Observatory (VAO) is hierarchical naming that combines the publishing organization name with the project or archive (e.g. "adil.ncsa") while leaving out fields like ".edu" or ".org". In the AstroGrid project, the convention is to use a DNS name in reverse order (e.g. "org.astrogrid.www"); this practice has the advantage of reducing the probability that two organizations will want to use the same authority identifier.

publishers, however, may support additional mappings between identifiers and other URIs (such as http URLs) that they manage; in this case, agents should only assume the mapping applies within the domain of the publisher.

\subsubsection{Authority}

A naming authority is an organization (usually a data provider) that has been granted the right by the IVOA to create IVOA-compliant identifiers for resources it registers. See sect. 3 for details on how this right is granted. The naming authority creates IVOIDs with empty local parts within the scope of one or more authority identifiers.

The authority component of an IVOID is severely restricted over RFC 3986 as follows:

- it MUST be at least three characters long

- it MUST begin with an alpha-numeric character

- it MUST NOT contain percent-encoded characters

- it MUST NOT contain characters outside of $\langle$ unreserved $\rangle$, with the tilde strongly discouraged

- there are no $\langle$ userinfo $\rangle$ or $\langle$ port $\rangle$ components

In ABNF, using the symbols from RFC 3986, an authority identifier in IVOIDs thus has the form:

$$
\langle\text { authority }\rangle=\langle\text { alphanum }\rangle\langle\text { unreserved }\rangle\langle\text { unreserved }\rangle *\langle\text { unreserved }\rangle
$$


A naming authority is allowed to control multiple authority identifiers to organize related resources into different namespaces. For example, an organization may choose to control two authority identifiers, one for research-related resources and one for education/outreach resources, even though they are all maintained by the same organization and perhaps made available through the same machine.

\section{Examples for valid authorities}

(1) nasa.heasarc

(2) n_1a.alph-0.02

(3) 123 (authorities can start with a number)

\section{Examples for invalid authorities}

(1) a2 (less than three characters)

(2) _temporary.id (authorities must begin with an alphanumeric character, which the underscore is not)

(3) DAT\%41 (percent-encoded characters are not allowed, even if they work out to be unreserved characters)

(4) de! uni-hd! physics\#ari (not entirely consisting of unreserved characters)

\subsubsection{Resource Key}

RFC 3986's $\langle$ path $\rangle$ part of an IVOID is called a resource key. It is a name for a resource that is unique within the namespace of an authority identifier. The naming authority creates keys for its namespaces and has complete control of their forms beyond the syntax constraints specified here.

On top of the definitions in RFC 3986 for paths, section 3.3, resource keys in IVOIDs are further constrained in that

- $\langle$ segment $\rangle$ MUST NOT contain percent-encoded characters

- $\langle$ segment $\rangle$ MUST NOT contain colons or commercial at signs

- Only $\langle$ path-abempty $\rangle$ expansions are allowed

In ABNF, using or overriding the symbols of RFC 3986, this means:

$$
\begin{aligned}
\langle\text { path }\rangle & =\langle\text { path-abempty }\rangle \\
\langle\text { segment }\rangle & =*\langle\text { ivo-segment-char }\rangle \\
\langle\text { ivo-segment-char }\rangle & =\langle\text { unreserved }\rangle /\langle\text { sub-delims }\rangle
\end{aligned}
$$

Naming authorities MUST NOT create path segments matching either "." or ".."; empty segments, resulting in two or more consecutive slashes or a trailing slash, are also forbidden. In particular, as described in sect. 2.6, such segments 
would not have the special meaning they have in traditional file system pathnames; that is, a resource key cannot be transformed by removing any kinds of segments and still reference the same resource.

Note that, as discussed in sect. 2.2, characters from 〈sub-delims〉 MUST NOT be used in resource keys unless their semantics is defined in an IVOA specification. As percent-encoded characters are not allowed in resource keys, these characters MUST NOT occur in generic Registry references at all.

The naming authority is free to create a resource key that suggests something about the resource it refers to. Any meaning that is suggested by the resource key is intended only for human consumption. The character content of a resource key is not semantically machine-interpretable within the context of the IVOA as defined by this document.

The presence of a resource key is optional. An identifier that contains only an authority identifier refers to the authority itself and MUST resolve to a vr: Authority-typed resource record (Plante et al., 2008) in the IVOA Registry.

VO applications MUST be case-insensitive when processing resource keys. In presentation, the preferred use of case is set by the rendering of the key by the naming authority when the IVOID is registered. This may contain capital letters to improve readability.

\section{Examples for valid resource keys}

(1) " " (i.e., the empty string; zero repetitions of ("/" 〈segment $\rangle)$ are legal)

(2) /reskey

(3) / - user/STScI_1/1a-7z.u (unreserved characters are allowed, and arbitrarily many segments are allowed)

\section{Examples of invalid resource keys}

(1) / (empty $\langle$ segment $\rangle$ s are forbidden)

(2) reskey (nonempty resource keys must always start with a slash)

(3) /data/ (empty $\langle$ segment $\rangle$ s are forbidden)

(4) /data//other (empty $\langle$ segment $\rangle \mathrm{s}$ are forbidden)

(5) $/$ data $/ \mathrm{c} / . . / \mathrm{d}(\langle$ segment $\rangle \mathrm{s}$ that indicate tree traversal in other URI schemes are forbidden)

(6) /data!g-vo.org (although this might become legal when some IVOA standard gives the bang - which is from $\langle$ sub-delims $\rangle$ - an extra meaning)

(7) / user $/ \mathrm{M} \%$ f cller (percent encoding is forbidden in resource keys; if it were, the codepoint $0 \mathrm{xfc}$ is not in $\langle$ ivo-segment-char $\rangle$; if that were true, it would still not be valid utf-8)

\subsubsection{Query}

This specification does not pose constraints on $\langle q u e r y\rangle$ beyond the definitions in RFC 3986. It also does not define any semantics.

Creators of IVOIDs are encouraged to adhere to URI semantics, i.e., IVOIDs with different query parts should refer to different resources. 
To allow some resilience towards clients erronerously case folding the query part, operators SHOULD NOT define IVOIDs referring to different resources differing only by case in the query part.

Still, operators are not required to perform case folding on query parts. Therefore, applications MUST NOT change the case of characters in query parts.

\section{Examples for valid query parts}

(1) par1=val1\&par2=val2 (the classic use for query parts in HTTP URLs as, e.g., generated by browser forms)

(2) //. .//! :?? (but sub-delims, slashes and question marks are allowed here, as are strings looking like forbidden segments in resource keys)

(3) $\% \mathrm{C} 2 \% \mathrm{~B} 5 \% 2 \mathrm{Her}$ (percent-encoding special characters is legal, but outside of ASCII one has to use utf- 8 ; this example works out to be " $m u$ Her")

(4) $\% 3 \mathrm{~A} \% 5 \mathrm{~B} \% 5 \mathrm{D}$ (while the generic delimiters \#, [, and ] are not allowed in query parts literally, they can be included in percent-encoded forms)

\section{Examples for invalid query parts}

(1) :\#[] bad (most generic delimiters are not allowed literally in query parts, nor is the blank)

(2) $\%$ B5\% $\%$ Her (sequences of percent-encoded characters must be valid utf- 8 after decoding)

\subsubsection{Fragment}

This specification does not pose constraints on $\langle$ fragment $\rangle$ beyond the definitions in RFC 3986.

Creators of IVOIDs are encouraged to adhere to URI semantics, i.e., fragment identifiers should be used to distinguish between different entities within the same parent resource as discussed in Gray (2012). The details of this process depend on the type of document being retrieved. See sects. 2.5 and 4.2 for details.

Applications MUST NOT change the case of characters in fragments.

For examples for valid and invalid fragments, see the examples for query parts in sect. 2.3.4

\subsection{Usage}

IVOIDs are used to identify resources in the general sense, i.e., they might refer to datasets, abstract concepts, etc.; their Registry parts, on the other hand, MUST always be dereferenceable, i.e., resolve in the VO Registry.

No hierarchy is implied in any of the components. Therefore, there are no relative URIs for IVOA Identifiers. In effect, this specification overrides the rule in section 4.1 of RFC 3986 to become 


$$
\langle U R I \text {-reference }\rangle=\langle U R I\rangle .
$$

\subsection{Reference Resolution}

Registry references can always be resolved to a Registry record by querying a searchable registry, for instance, using RegTAP (Demleitner et al., 2013). Clients will usually have some Registry endpoint URLs built in, more are discoverable as described in Benson et al. (2009). In a full registry with an OAIPMH interface, the OAI-PMH GetRecord operation provides another means for obtaining the Registry record referenced by an IVOID.

If an IVOID's Registry part does not resolve in the Registry, clients SHOULD assume it is obsolete and that any IVOID built with it does not reference an existing resource or entity either.

When dereferencing IVOIDs with query parts, applications should first dereference the reference part to a registry record. From that, a service should be identified that can dereference the full IVOID. Concrete procedures may be given in IVOA specifications introducing certain resource types. One example for this is sect. 4.1.

There is no mechanism that would allow applications to tell from an IVOID's form whether or not it can be dereferenced in any special way. Any such information has to be obtained from the context the IVOID is found in.

For resolving IVOIDs with fragment identifiers, applications would again resolve the Registry part in the Registry. In the presence of a query component, it would be dereferenced as just discussed to obtain a basic document, otherwise the basic document is the Registry record itself. The entity referred to is then extracted from the basic document by means specific to the document type; one example of such a prescription is given in sect. 4.2.

As there are no relative IVOIDs, most of RFC 3986's section 5 does not apply here.

\subsection{Normalization and Comparison}

An important use of identifiers is comparing two instances to determine if they refer to the same resource. This will most commonly occur when using an identifier to look up the associated resource description in a registry.

IVOID comparison is according to RFC 3986, section 6.2.2, with the following additional regulations:

- As no hierarchy is implied in any IVOID part, no path segment normalization is ever performed on IVOIDs.

- As IVOIDs must not percent-encode characters that do not need to be encoded, no percent-encoding normalization is ever performed on IVOIDs. 
- In addition to scheme and authority as in RFC 3986, in IVOIDs the resource key is also compared case-insensitively. This means that Registry references can be case-folded for processing.

Note that neither query parts nor fragment identifiers may be compared case-insensitively or normalized in any other way; allowing this would severely impact their usefulness, as they, in general, refer to case-sensitive entities like XML ids or file system paths.

No further normalizations are performed in IVOID comparison, i.e., sections 6.2.3 and 6.2.4 of RFC 3986 do not apply.

For instance, given the IVOID

$$
\text { ivo://example.com/res/key1?par=U\%20Pic\#Part1, }
$$

the IVOID

$$
\text { IVO://EXAMPLE. COM/RES/KEY1?par=U\%20Pic\#Part1 }
$$

must compare equal, while the following IVOIDs must compare non-equal:

- ivo://example.com/res/key1?par=u\%20Pic\#part1 (query part and fragment are non case-insensitive)

- ivo://example.com/./res/key1?par=U\%20Pic\#Part1 (no path normalization takes place, even if that were a legal IVOID)

- ivo://example.com/res/key1?par=U\%20Pic (fragment identifiers may not be stripped off for comparison)

- ivo://example.com/res/key1?par=U\%20Pic\&\#Part1 (query parts are not parsed, and their interpretation as key/value pairs is up to data providers)

- ivo://example.com/res/\%6Bey1?par=U\%20Pic\#Part1 (no normalization of percent encoding takes place)

In general, the string-based comparison of identifiers cannot determine definitively if two identifiers refer to different resources. While it is not intended that a Registry record is registered multiple times with different identifiers, it is not disallowed by this specification. In particular, it is possible that two resources with different identifiers may be mirrors of each other; such a relationship can only be determined by examining the metadata contained in the descriptions associated with each identifier.

This concludes the additional constraints and regulations for IVOIDs over RFC 3986 compliant URIs. The remainder of this document standardizes certain aspects not in the scope of RFC 3986. 


\section{Creating Identifiers}

An important aim of the process for creating identifiers is to ensure uniqueness. In the context of IVOA identifiers, "unique" means that a given identifier MUST NOT refer to two different resources at any instant. Furthermore, the identifier SHOULD refer to at most one resource over all time; that is, IVOIDs should not be reused for unrelated resouces. Note that a resource may potentially be dynamic (such as 'weather at telescope' or 'current version of the standard') here, there is a conceptually unique resource, even though the content of it may change in time.

Another aim of the identifier creation process is to trace the delegation of authority over the identifier. In practice, a Registry reference is created by an organization when registering a resource. Thus, only recognized naming authorities (or persons representing such organizations) may create Registry references.

The details of the service used to claim a naming authority is described in the IVOA Registry Interfaces standard (Benson et al., 2015).

Once an organization is recognized as a naming authority, it is free to register any number of resources with identifiers having an authority identifier that they control. No organization may create an identifier with an authority identifier it does not control. The naming authority has full control over the creation of a resource key as long as it conforms to the syntax and uniqueness constraints described in this specification.

Likewise, once a Registry reference is established, any number of IVOIDs may be built using it (e.g., when publishing new datasets). In this case, the VO Registry is not involved, IVOID creation happens under the exclusive control of the owner of the service or data collection the Registry reference refers to.

\section{Special Identifier Types}

This section discusses some special classes of IVOIDs that reference something other than Registry records and for which identifier forms for one reason or other must or should be uniform across the different other standards that define the resources referenced.

\subsection{Dataset Identifiers}

DAL standards like Obscore (Louys et al., 2011), SSAP (Tody et al., 2012), or Datalink (Dowler et al., 2015) need to reference datasets. The SSAP standard defines these as "an individual data object usually including associated metadata." In astronomy, single images or spectra are datasets, but tables or more complex data products might, at the publisher's discretion, also be referenced as a single dataset. 
A reference to a dataset is called a dataset identifier (DID), more specifically publisher DID if the DID was assigned by the dataset's publisher, and creator DID if the DID was assigned by the dataset's author. Various standards mandate that DIDs must be IVOIDs.

Historically, DIDs were customarily formed by adding fragment identifiers to Registry reference, a practice recommended in SSAP in versions up to 1.1. This definition was criticized in Gray (2012) as a potential interoperability issue.

Therefore, this specification deprecates the regulation from SSAP 1.1. Instead, DIDs in the VO now MUST use the query part to distinguish datasets within one VO resource. In short, the separator between Registry reference and local part now must be the question mark rather than the octothorpe. A welcome side effect is that the fragment identifier can now be used to reference sub-entities within the datasets.

An example for a dataset id (that should actually resolve according to the scheme laid out below) is

ivo://org.gavo.dc/ ?flashheros/data/ca92/f0065.mt.

Existing DIDs in services implementing SSAP up to 1.1 and Obscore 1.0 are not affected by these requirements and may be used until the respective services are updated to newer standards.

Note that by this specification publishers have no obligation to ensure continued access to datasets identified with PubDIDs. They are not by themselves persistent identifiers with guarantees on resolvability. Their main function is to provide globally unique identifiers for use in, e.g., federating responses from different services.

Publishers are, however, encouraged to declare at least one capability of a protocol dealing with PubDIDs ${ }^{2}$ in the resource record referenced by the Registry part of a PubDID (i.e., the URI in front of the first question mark). In that way, clients can attempt to retrieve data based on stand-alone PubDIDs by querying the Registry for the "embedding" resource and seeing if it supports any protocol they implement.

The definition of a proper resolver or resolution strategy is beyond the scope of this standard. Although services prototyping such funtionality have been written $^{3}$, we maintain additional efforts are required outside of Registry to build a reliable infrastructure on top of PubDIDs.

\subsection{Standard Identifiers}

In many VO standards, it is important to express adherence to a set of constraints. Common examples include the declaration of the protocol - and the version of the protocol - that an endpoint implements in VOResource's

\footnotetext{
${ }^{2}$ At the time of this writing, Datalink, Obscore, and SSA are IVOA recommended protocols allowing queries involving PubDIDs. SIA (Tody and Plante, 2009) will, according to current proposed recommendations, have an analogous facility in version 2.0.

3 e.g., GAVO's global PubDID resolver at http://dc.g-vo.org/glopidir.
} 
capability element or a data model represented with a TAP service in TAPRegExt. The resource record such identifiers reference is defined by StandardsRegExt (Harrison et al., 2012). As such records typically describe multiple versions of a standard, and a single standard may contain definitions of multiple different capabilities that need to be discerned, the simple Registry Reference of the standard record usually is not enough.

Therefore, StandardsRegExt records should define one key element for each such referenceable entity. The name child of this key, denoting both the kind of capability and the major and minor version, is then what is referenced by the identifier as defined by StandardsRegExt, such that the complete element will typically have the form

$$
\langle\text { standard-ref }\rangle \text { "\#" }\langle\text { key-name }\rangle \text { "-" }\langle\text { version }\rangle
$$

For instance, the standard exampleProto might define both a data model model and a query capability query. In its version 1.0, there would be two standard keys model-1.0 and query-1.0. In a capability element in another resource's Registry record, support of the query capability would then be declared with the IVOID ivo://ivoa.net/std/exampleProto\#query-1.0, whereas a TAP service exposing the model would contain a dataModel element with an ivo-id attribute of ivo://ivoa.net/std/exampleProto\#model-1.0.

As the exampleProto develops, new standard keys like query-1.1 or query-2.0 are added. Note that while ideally, the version tags in the keys will correspond to the version of the document that defines them, this is not a requirement. Indeed, if the underlying model has no incompatible changes, even exampleProto 2.0 might specify that its data model would remain ivo://ivoa.net/std/exampleProto\#model-1.0. This allows clients to easily discover all services they can operate.

Registry interfaces will typically offer some pattern matching capability for comparing such identifiers. Clients should use that feature to ignore minor versions if appropriate - by the IVOA's versioning rules (Hanisch et al., 2010), a generic client for version 1 of a protocol should be able to operate all version 1 services, regardless of their minor versions, and clients implementing multiple versions of a standard can entirely ignore the version tag. For instance, with RegTAP (Demleitner et al., 2013) an exampleProto 1.0 client would look for capabilities for which

standard_id LIKE 'ivo://ivoa.net/std/exampleProto\#query-1.\%'

holds, whereas a client that speaks both versions 1 and 2 of the protocol would look for capabilities with

standard_id LIKE 'ivo://ivoa.net/std/exampleProto\#query-\%'.

\section{A Changes from Previous Versions}




\section{A.1 Changes from PR-2015-07-09}

- Now deprecating the term IVORN, as historical usage has been too inconsistent. Instead, there is now the "Registry part" of an IVOID, and an IVOID that only has a registry part is called a Registry reference.

- More examples

- No longer suggesting a concrete algorithm for PubDID resolution; instead, clear encouragement to PubDID minters to point to appropriate services from the Registry part of a PubDID.

- Editorial changes

\section{A.2 Changes from 1.12}

- Removed the (unused) XML representation of Identifiers.

- Rewrote the section on URI forms to more closely correspond to the organization of RFC 3986.

- Case-insensitive handling of IVORNs is now a MUST.

- Now allowing percent-encoded items outside of the authority and resource key.

- Added rules for forming URI-compliant dataset identifiers

- Added rules for forming StandardsRegExt-compliant standard identifiers.

- Empty path segments, as well as those consisting exclusively of dots, are now forbidden rather than just discouraged.

- Dropped the recommendation to present authority identifiers in lower case.

- Generally moved to IVOID as the abbreviation for IVOA identifier, defined IVORN to be the part of an IVOID without a local part.

- Removed some obsolete introductory material that has been superseded by other standards.

- Migrated to ivoatex source

\section{A.3 Changes from v1.10}

- Moved "!" from the discouraged list of characters to the reserved list, thereby disallowing its inclusion in IVOA identifiers.

- Clarified the list of characters disallowed in an authority ID by:

- explicitly disallowing URI-escaped sequences. 
- listing as reserved characters only those characters that are allowed by the URI spec but disallowed by this one.

- Listed in a tip box the characters that are disallowed by the URI spec.

As before, the definition of the resource key refers to the same list of reserved characters as those disallowed.

- Fixed numerous links and references.

\section{A.4 Changes from v1.0}

- The prohibition of using "+" and "=" within Identifier components has been dropped.

- Recommendations for authority ID strings have been updated to match current practice in AstroGrid and the NVO.

- In the example schema in App. A, the namespace was altered to conform with IVOA conventions. A correction was also made to the allowed pattern for AuthorityIDType to properly comply with the XML specification defined in section 3.2.1.

- various clarifications based on reviewer comments

\section{A.5 Changes from v0.1}

- Resource key is now required except when referring to a naming authority itself.

- support for DNS-like authority IDs clarified.

- added role of \# and ? as "stop" characters in URI form.

- dropped non-binding Appendix B: Recommended Mechanism for becoming a Naming authority.

\section{References}

Arviset, C., Gaudet, S. and the IVOA Technical Coordination Group (2010), 'IVOA architecture', IVOA Note.

URL: http://www.ivoa.net/documents/Notes/IVOAArchitecture

Benson, K., Plante, R., Auden, E., Graham, M., Greene, G., Hill, M., Linde, T., Morris, D., O’Mullane, W., Rixon, G., Stébé, A. and Andrews, K. (2009), 'IVOA registry interfaces version 1.0', IVOA Recommendation.

URL: http://www.ivoa.net/documents/RegistryInterface/ 
Benson, K., Plante, R., Auden, E., Graham, M., Greene, G., Hill, M., Linde, T., Morris, D., O'Mullane, W., Rixon, G., Stébé, A. and Andrews, K. (2015), 'IVOA registry interfaces version 2.0', IVOA Recommendation.

URL: http://www.ivoa.net/documents/RegistryInterface/

Berners-Lee, T., Fielding, R. and Masinter, L. (2005), 'Uniform Resource Identifier (URI): Generic syntax', RFC 3986.

URL: http://www.ietf.org/rfc/rfc3986.txt

Bradner, S. (1997), 'Key words for use in RFCs to indicate requirement levels', RFC 2119.

URL: http://www.ietf.org/rfc/rfc2119.txt

Crocker, D. and Overell, P. (1997), 'Augmented BNF for syntax specifications: ABNF', RFC 2234.

URL: http://www.ietf.org/rfc/rfc2234.txt

Demleitner, M., Harrison, P., Molinaro, M., Greene, G., Dower, T. and Perdikeas, M. (2013), 'IVOA registry relational schema', IVOA Working Draft.

URL: http://www.ivoa.net/documents/RegTAP/

Dowler, P., Bonnarel, F., Michel, L. and Demleitner, M. (2015), 'Datalink', IVOA Recommendation.

URL: http://www.ivoa.net/documents/DataLink/

Gray, N. (2012), 'URI fragments in IVOA specifications', IVOA Note.

URL: http://www.ivoa.net/documents/Notes/URIFragments

Hanisch, R., IVOA Resource Registry Working Group and NVO Metadata Working Group (2007), 'Resource metadata for the virtual observatory', IVOA Recommendation.

URL: http://www.ivoa.net/documents/latest/RM.html

Hanisch, R. J., Arviset, C., Genova, F. and Rino, B. (2010), 'IVOA document standards, version 1.2', IVOA Recommendation.

URL: http://www.ivoa.net/documents/DocStd/

Harrison, P., Burke, D., Plante, R., Rixon, G. and Morris, D. (2012), 'StandardsRegExt: a VOResource schema extension for describing IVOA standards, version 1.0', IVOA Recommendation.

URL: http://www.ivoa.net/documents/StandardsRegExt/20120508/RECStandardsRegExt-1.0-20120508.html

Louys, M., Bonnarel, F., Schade, D., Dowler, P., Micol, A., Durand, D., Tody, D., Michel, L., Salgado, J., Chilingarian, I., Rino, B., de Dios Santander, J. and Skoda, P. (2011), 'Observation data model core components and its implementation in the Table Access Protocol, version 1.0', IVOA Recommendation. 
URL: http://www.ivoa.net/documents/ObsCore/20111028/REC-ObsCorev1.0-20111028.pdf

Plante, R., Benson, K., Graham, M., Greene, G., Harrison, P., Lemson, G., Linde, T., Rixon, G. and Stébé, A. (2008), 'VOResource: an XML encoding schema for resource metadata version 1.03', IVOA Recommendation.

URL: $\quad h t t p: / / w w w . i v o a . n e t / d o c u m e n t s / R E C / R e R / V O R e s o u r c e-$ 20080222.html

Tody, D., Dolensky, M., McDowell, J., Bonnarel, F., Budavari, T., Busko, I., Micol, A., Osuna, P., Salgado, J., Skoda, P., Thompson, R. and Valdes, F. (2012), 'Simple spectral access protocol version 1.1', IVOA Recommendation. URL: $\quad h t t p: / / w w w . i v o a . n e t / d o c u m e n t s / S S A / 20120210 / R E C-S S A-1.1-$ 20120210.htm

Tody, D. and Plante, R. (2009), 'Simple image access specification', IVOA Recommendation.

URL: http://www.ivoa.net/documents/latest/SIA.html 